\title{
P I 9-57 LB. Gene-based vaccination protects against mucosal infection by a heterologous highly pathogenic SIV isolate in rhesus
} monkeys

\author{
N Letvin'1, J Mascola*2, S Rao ${ }^{2}$, A Buzby' ${ }^{1}$ M Roederer ${ }^{2}$, M Hudgens ${ }^{3}$, \\ P Gilbert ${ }^{4}$, R Seder ${ }^{2}$, D Douek², R Koup², B Graham² and G Nabel ${ }^{2}$
}

\author{
Address: ${ }^{1}$ Beth Israel Deaconess Hospital, Harvard Medical School, Boston, MA, USA, ${ }^{2}$ Vaccine Research Center, NIAID, NIH, Bethesda, MD, USA, \\ ${ }^{3}$ University of North Carolina at Chapel Hill, Chapel Hill, NC, USA and ${ }^{4}$ Fred Hutchinson Cancer Research Center, Seattle, WA, USA \\ * Corresponding author
}

from AIDS Vaccine 2009

Paris, France. 19-22 October 2009

Published: 22 October 2009

Retrovirology 2009, 6(Suppl 3):P42 I doi:I0.I I86/I742-4690-6-S3-P42 I

This abstract is available from: http://www.retrovirology.com/content/6/S3/P42I

(C) 2009 Letvin et al; licensee BioMed Central Ltd.

\section{Background}

A goal of an AIDS vaccine is to prevent acquisition of HIV1 infection. The ability of AIDS vaccine candidates to protect against mucosal infection by heterologous viruses in non-human primates will help to define mechanisms and correlates of immune protection relevant to humans.

\section{Methods}

Rhesus monkeys were immunized intramuscularly using a plasmid DNA prime/recombinant Ad5 boost regimen. Experimental animals received vectors expressing genes encoding SIVmac239 env and gag/pol, and control animals received empty vectors. Monkeys were challenged 4 months after their final immunization with a series of 12 weekly intra-rectal exposures of SIVsmE660 in one study, and SIVmac251 in a second study. The challenge dose for each virus was titered to achieve $50 \%$ infection per-inoculation for control animals. Time to acquisition of infection was monitored by weekly assessment of plasma SIV RNA levels.

\section{Results}

Experimentally vaccinated monkeys were significantly protected against infection by the heterologous virus, SIVsmE660 ( $\mathrm{n}=25$ per arm). There was a 50\% reduction in acquisition of infection and a significant increase in time to infection in vaccinated animals as compared to the control animals (log-rank test, $\mathrm{p}=0.0009$ ). There was no significant difference in peak viral loads of infected vaccinated animals compared to infected controls. Immune correlates of vaccine protection are being evaluated. A similar vaccine regimen revealed no protection from acquisition of infection when homologous SIVmac251 was used as the challenge virus, and effects on viral load seen previously with this virus are under investigation.

\section{Conclusion}

This study demonstrates vaccine protection against mucosal acquisition of a heterologous, pathogenic SIV isolate in rhesus monkeys. The absence of protection against acquisition of another pathogenic SIV isolate illustrates the biological variability of primate immunodeficiency viruses. Protection in a heterologous challenge model will permit definition of immune correlates of protection and facilitate the development of highly effective AIDS vaccines. 